\title{
A Comprehensive Model for Liquid Film Boiling in Internal Combustion Engines
}

\author{
C. Habchi \\ Institut français du pétrole, IFP, 1-4 avenue de Bois-Préau, 92852 Rueil-Malmaison Cedex - France \\ e-mail: chawki.habchi@ifp.fr
}

\begin{abstract}
Résumé - Un modèle complet pour l'ébullition de film liquide dans les moteurs à combustion interne - Dans cet article, les principaux processus physiques, régissant les régimes d'ébullition nucléée et de transition d'un film liquide, ont été examinés à partir des observations expérimentales disponibles dans la littérature. Les tendances physiques observées, ont été utilisées, pour développer un modèle phénoménologique complet pour l'ébullition de film liquide (LFB). Celui-ci permet le calcul de sa vaporisation, dans le régime d'ébullition nucléée, ainsi que, dans le régime d'ébullition de transition. Ces régimes sont identifiés par les températures de saturation, de Nukiyama et de Leidenfrost. L'estimation des températures de Nukiyama et de Leidenfrost, en fonction de la pression de gaz ambiante, a requis une attention toute particulière.

Plusieurs courbes de durée de vie, de gouttes de grosse taille déposées sur une surface chaude dans diverses conditions, ont été choisies parmi celles qui sont disponibles dans la littérature récente pour la validation du modèle LFB. Les résultats numériques montrent que les ordres de grandeur et les tendances observées expérimentalement sont bien respectés. En particulier, le modèle LFB reproduit bien la disparition progressive du régime de Leidenfrost avec des pressions suffisamment élevées du gaz. D'autre part, l'augmentation progressive du taux de vaporisation du liquide avec la rugosité du mur, précédemment observée expérimentalement près du point de Leidenfrost, a été correctement prédite par le modèle LFB.
\end{abstract}

\footnotetext{
Abstract - A Comprehensive Model for Liquid Film Boiling in Internal Combustion Engines - In this paper, the main physical processes governing the nucleate and transition regimes of the boiling of a liquid film were reviewed from the available experimental observations in the literature. The physical tendencies observed in most experiments have been used to develop a comprehensive phenomenological Liquid Film Boiling (LFB) model which allows the calculation of the vaporization of liquid films in the nucleate boiling regime as well as in the transition boiling regime. These regimes are identified by the temperatures of saturation, Nukiyama and Leidenfrost. A particular attention has been made concerning the estimation of Leidenfrost and Nukiyama temperatures as a function of the ambient gas pressure. Several curves of lifetime of rather bulky droplets deposited on a hot surface under various conditions and chosen among those which are available in the recent literature have been used for the validation of the LFB model. The numerical results show that the orders of magnitude and the tendencies observed experimentally are well respected. Particularly, the LFB model reproduces well the progressive disappearance of the Leidenfrost regime observed in experiments with sufficiently high gas pressures. In addition, the gradual increase of the vaporization rate with wall roughness which was previously observed experimentally near the Leidenfrost point has been correctly predicted by the LFB model.
} 


\section{INTRODUCTION}

In automobile engines, the technology of direct injection in the combustion chamber is used more and more because it allows controlling the consumption of combustible better in comparison to port fuel injection engines. Besides, the pressures used in the injection systems are increased more and more to improve the spray atomization, mixture preparation and combustion. In these conditions, liquid spray may impinge the internal surface of the combustion chamber. This interaction of spray with the wall involves different physical phenomena according to the conditions of impact (speed of the droplets, temperature of the wall, roughness of the surface, etc.) and of the pressure of gases in the combustion chamber [1-7].

In diesel engines for instance, injection is often made in the course of the compression of fresh gases. As the saturation temperature of fuel oil increases with the pressure of gases, the impact of spray on the wall leads in most cases to the formation of a liquid film. The latter evaporates rather slowly and may even survive to the combustion [8]. In the course of the expansion stroke, the saturation temperature of fuel oil diminishes with gas pressure to the point of attaining a value lower than the wall temperature and consequently leading to the boiling of the liquid film. Furthermore, the Leidenfrost and Nukiyama temperatures diminish as well during the expansion stroke leading to a continuous variation of the boiling regimes. Very few works in the literature were dedicated to model these phenomena for shallow liquid films. Based on available experimental observations in the literature, this paper proposes a synthetic vision of the physical processes taking place during the different modes of boiling of a thin liquid film. The physical tendencies observed in most cases with experiments of type "pool boiling" and "sessile droplet" or deposited droplet on a hot wall, are discussed in the first section. Next, the identified main physical parameters of the boiling process are modeled as functions of the wall temperature. The equations of the Liquid Film Boiling (LFB) model are then presented. The third section is dedicated to the LFB model validation. A particular attention is paid to the estimation of Leidenfrost and Nukiyama temperatures. Finally, experimental lifetime curves of droplets having diameters larger than $2 \mathrm{~mm}$ and which were deposited on a hot wall were used to validate the LFB model in different conditions including the variations of wall roughness [9] and ambient gas pressure [10].

\section{PHYSICS OF BOILING}

Among the physical processes occurring when a liquid film is formed on the surface of a combustion chamber of a direct injection engine, heat transfer and phase changes are of primary importance. The vaporization rate of the liquid film is not only a function of the wall temperature but it is strongly affected by the gas pressure variation inside the combustion chamber. Indeed, processes occurring during the piston expansion stroke are similar to those occurring when increasing the wall temperature $T_{w}$. In this last case, one may distinguish three or four regimes or modes of vaporization in the literature $[1,11,12]$, which can be classified according to the extent of superheating of the wall $\Delta T_{w s a t}=\left(T_{w}-T_{\text {sat }}\right)$ and using as limits for these regimes, the saturation temperature $T_{\text {sat }}$, the Nukiyama temperature $T_{N}$ and the Leidenfrost temperature $T_{L}$. The two last critical temperatures are usually determined experimentally from the boiling or lifetime curve of a droplet (Fig. 1). This curve is obtained by measuring the total time that it takes a droplet to completely evaporate after it has been gently deposited on a hot wall [13-15]. The different boiling regimes are discussed in the following paragraphs in order to emphasize the data available for the modeling of the boiling processes and their validation.

\section{Mode I, the complete wetting regime when $T_{w}<T_{\text {sat }}$}

The spray, impinging a wall having a temperature $T_{w}$ smaller than the saturation temperature, forms a liquid film which evaporates slowly. In this mode, the evaporation rate strongly depends on the turbulence level in the ambient gas [16-18].

Mode II, the nucleate boiling regime when $T_{s a t}<T_{w}<T_{N}$ In this regime, the liquid very close to the wall (e.g. in the thermal boundary layer) is overheated. This leads to the formation of vapor cavities which are born starting from germs (or sites of nucleation) often hidden in the wall roughness. While growing, these cavities are detached and form small bubbles. This process of phase change prevents the temperature of the liquid $T_{l}$ from exceeding its temperature of saturation by consuming most of the heat flux $Q_{w l}$ transferred to the liquid by the wall. The experimental observations of Cornwell and Brown and Singh et al. (quoted in the review

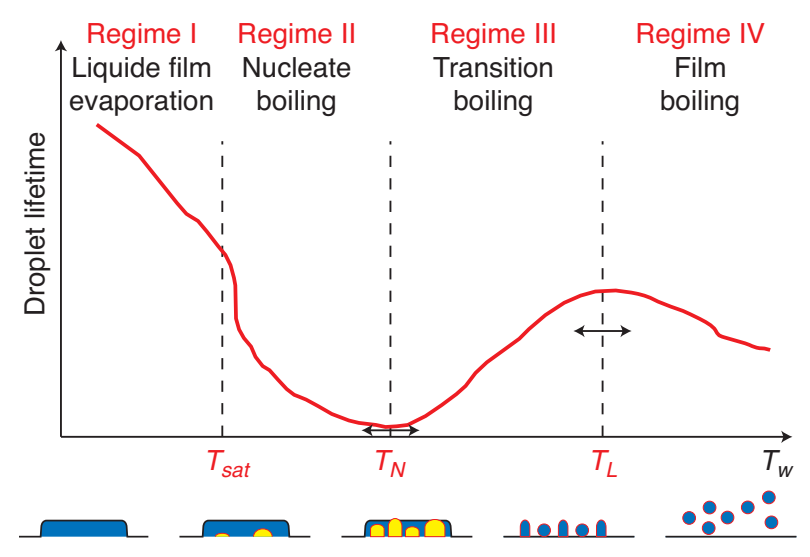

Figure 1

Droplet lifetime curve and schematics illustrating the boiling states near the limits of the boiling regimes. 
of Dhir [12]) showed that in the nucleate boiling case of water under atmospheric conditions, the number of vapor cavities $N c$ is at the same time proportional to $\Delta T_{w s a t}^{2}$ and inversely proportional to the square of the diameter of the cavities, $D_{c}^{2}$. Thus, the bubbles formed have four main effects:

- They increase the dry fraction of the wall, $\alpha_{d r y}$ :

$$
\alpha_{d r y}=\frac{A_{d r y}}{A_{w}}=\frac{\sum_{i=1}^{N c} \frac{\pi D_{c}^{2}}{4}}{A_{w}}
$$

where $A_{w}$ is the total wetted surface in the absence of boiling. The value of $\alpha_{d r y}$ which corresponds to the minimal lifetime duration of a droplet (defined when $T_{w}=T_{N}$ ) was estimated by several authors in order to calculate the boiling curve and in particular the Critical Heat Flux (CHF). The measured value of the dry fraction by Iida and Kobayasi [19] is $\alpha_{d r y}\left(T_{w} \approx T_{N}\right) \approx 0.9$ which is approximately the same as the value obtained by the model of Nishio and Tanaka [20]. One can also get an estimate of $\alpha_{d r y}$ by using Equation (1). Indeed, assuming that the maximum value of the cavities diameter $D_{c}$ is attained when $T_{w}=T_{N}$ (i.e. just before the beginning of the collapse of the cavities):

$$
\alpha_{d r y}\left(T_{w} \approx T_{N}\right)=\frac{\pi}{4} \approx 0.8
$$

has been obtained [20]. Furthermore, Dhir and Liaw [21] found that during pool boiling, $\alpha_{d r y}\left(T_{w} \approx T_{N}\right)$ varies between 0.68 and 0.8 when contact angles vary between 27 and 90 degrees respectively.

- They accelerate the evaporation of the liquid at the wall. Indeed, several authors noted this acceleration. Dhir [12] has noticed that in the case of nucleate boiling of water, most of the evaporation is carried out in the periphery of the vapor cavities close to the contact lines. The length density of these contact lines is then a parameter of great importance as far as nucleate boiling is concerned. This parameter (referred to below as $C_{l l d}$ ) corresponds to the sum of the perimeters of the cavities or dry zones.

$$
C_{l l d} \approx \sum_{i=1}^{N c} \pi D_{c_{i}}
$$

While following the same reasoning, Nishio and Tanaka [20] considered that the CHF point and the point of minimum lifetime $\left(T_{w}=T_{N}\right)$ correspond to the same conditions for which contact line length density is maximum. This maximum contact line length density value (denoted $C_{\text {lld }}^{\max }$ ) was found close to $3000 \mathrm{~m} / \mathrm{m}^{2}$ at $T_{w}=T_{N}$. As a matter of fact, $C_{l l d}$ increases between $T_{s a t}$ and $T_{N}$, then decreases between $T_{N}$ and $T_{L}$ in a way similar to the boiling curve. It worth noting that Nishio and Tanaka have used an optical technique to visualize the liquid-wall contact zones and the boiling structures for high heat-flux pool boiling conditions. The test liquid was R141b at atmospheric pressure and the boiling surface is a sapphire plate coated with an electro-conductive film. Although these experimental conditions are not really representative of those encountered in piston engines, the paper of Nishio and Tanaka [20] is the only reference that the author has found in the literature which provides experimental values of $C_{l l d}$.

- In the case of an horizontal wall, the bubbles can go up towards the liquid-gas interface. In this case, the collapse of these bubbles at the liquid surface leads to the production of tiny droplets which are ejected in the gas and which are characteristic of this boiling regime. Dhir [11] also underlines that the bubbles act as a pump which moves away the hot liquid from the wall. These phenomena are important in the case of "pool boiling" and do not seem to be important for shallow liquid films which are of interest in this study.

- They cool the wall by pumping the latent heat of vaporization necessary for the phase change especially when the temperature of gas is lower than the temperature of the wall. This process is of course desired for the cooling technologies. Indeed, most of the references cited previously have aimed at better understanding the CHF conditions in order to optimize cooling systems.

\section{Mode III, the transition boiling regime $\left(T_{N}<T_{W}<T_{L}\right)$}

In this regime, the small cavities on the wall and the bubbles may coalesce and form vapor columns and/or a larger vapor pocket. Several authors (see [11, 20, 22]) explained the decrease of the heat flux provided by the wall and the increase of the droplet lifetime, by the coalescence between them of the vapor cavities on the wall. This process can be regarded as the initial phase of the subsequent Leidenfrost regime during which a vapor cushion completely prevents the contact of the liquid with the wall. As a consequence, in this transition boiling regime, the heat flux passes more and more through a vapor cushion between the wall and the liquid, and therefore strongly increases the droplet lifetime.

\section{Mode IV, the Leidenfrost regime when $T_{w}>T_{L}$}

This regime which is often called "film boiling regime" is the result of intense evaporation which leads to the formation of a vapor cushion and prevents the contact of the liquid with the wall as just said above. The lifetime curve (Fig. 1) shows that the evaporation rate in this regime is less intense than in the preceding boiling regimes. This observation is all the more true as the ambient gas pressure is low, particularly when it is lower than 1 bar. Indeed, Temple-Pediani [23] and more recently Stanglmaier et al. [10] studied the effect of the ambient gas pressure on the evaporation of a droplet posed on a hot wall. They showed that the Leidenfrost regime is less and less important as the gas pressure increases. Furthermore, the experimental visualizations of Chaves et al. [2] and Moita and Moreira [7] show a cloud of droplets above the wall, which 
levitates under the effect of the Stefan flow induced by the evaporation. Therefore, in this boiling regime, the wall heats the vapor cushion which in its turn heats the liquid. The heat flux can be written in this case as follows:

$$
Q_{w v l}=\lambda_{v} \frac{\left(T_{w}-T_{l}\right)}{\delta_{v}}
$$

where $\lambda_{v}$ and $\delta_{v}$ are respectively the thermal conductivity of the vapor and the thickness of the vapor cushion. In addition, Fardad and Ladommatos [9] underlined the influence of the nature of the wall (roughness, adsorption, wettability, color, type of metal, etc.) on the Leidenfrost regime. In particular, they observed that roughness decreases the lifetime duration of the droplet. They explained this phenomenon by saying that the peaks of roughness contribute to increase the heat flux by wall-liquid direct contact and thus lead to a larger evaporation rate (Fig. 2). Moreover, the increasing importance of the wall roughness with surface temperature has been underlined by Moita and Moreira [7]. From their experiments on the disintegration mechanism of impinging droplets onto heated rigid surfaces in the Leidenfrost regime, they also reported that the wall roughness is as important as the dynamic vapor pressure (i.e. the Stephan flow) associated with the rate of vaporization.

\section{LIQUID FILM BOILING MODEL}

According to the experimental evidence summarized above, the intensity of the boiling phenomenon seems to be governed by two main parameters:

- the dry fraction of liquid film area due to boiling $\alpha_{d r y}$;

- the length of the contact lines of the liquid film $C_{l l d}$.

The new model of Liquid Film Boiling (referred to below as LFB) which is presented in this paper is based on different phenomenological correlations. The two first describe the behaviour of the above parameters according to each boiling regime.

First, two dimensionless functions have been defined: the function for the dry fraction $\alpha_{d r y}$ and the function for the length density of the contact lines of the liquid film, $k_{\text {clld }}$. The way according to which these functions were obtained is given below in Sections 2.1 and 2.2, respectively.
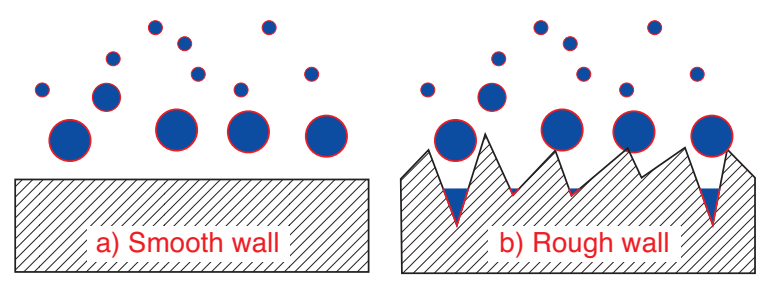

Figure 2

Schematics illustrating how the surface roughness promotes the liquid-wall contact in the Leidenfrost regime.

\subsection{Function of Dry Fraction}

The dry fraction $\alpha_{d r y}$ is defined by Equation (1). It varies inevitably between 0 and 1 when the wall temperature increases from the saturation temperature to the Leidenfrost temperature respectively. Moreover, the experimental observations mentioned in Section (1) showed that $\alpha_{d r y}$ increases quickly during the nucleate boiling regime. It reaches a value of about 0.7 to 0.9 when $T_{w}=T_{N}$. This behavior of the function $\alpha_{d r y}\left(T_{w}\right)$ has been expressed by the following relation:

$$
\alpha_{d r y}\left(T_{w}\right)=\alpha_{d r y}^{P L} T_{*}^{1 / 4}
$$

where $T_{*}$ is defined by:

$$
T_{*}=\frac{T_{w}-T_{s a t}}{T_{L}-T_{s a t}}
$$

and $\alpha_{d r y}^{P L}$ is the value of $\alpha_{d r y}$ at the Leidenfrost point (i.e. when $T_{w}=T_{L}$ ). A value of $\alpha_{d r y}^{P L}=0.98$ is used as we considered that the liquid takes off completely from the wall shortly after the Leidenfrost point.

\subsection{Function of Length Density of Contact Lines}

In the nucleate regime of boiling, the length of contact lines increases as the bubbles diameters increase at the wall surface. Hence, the function of length density of contact lines (referred to below as $C_{l l d}\left(T_{w}\right)$ ) will be assumed to follow the same evolution as the function of dry fraction $\alpha_{d r y}\left(T_{w}\right)$. The maximum value of $C_{l l d}\left(T_{w}\right)$ function is assumed to take place at the Nukiyama point (i.e. when $T_{w}=T_{N}$ ). In these conditions, Nishio and Tanaka [20] have measured values more or less equal to $C_{l d}^{\max } \approx 3000 \mathrm{~m} / \mathrm{m}^{2}$. Then, in the transition regime of boiling, $C_{l l d}\left(T_{w}\right)$ decreases towards a minimal value at the Leidenfrost point (i.e. when $T_{w}=T_{L}$ ), by effect of coalescence of the various nucleation cavities and by the progressive formation of a vapor cushion (see the corresponding diagrams in Fig. 3). This minimal value $C_{l l d}\left(T_{L}\right)$ depends on the nature of the wall and its roughness in particular. To model this behavior according to the temperature of the wall, a dimensionless function of length density of contact lines $k_{\text {clld }}$ is defined in the following way:

$$
k_{\text {clld }}\left(T_{w}\right)=\frac{C_{l l d}\left(T_{w}\right)}{C_{l l d}^{\max }}
$$

This function increases from 0 to 1 during the nucleate regime of boiling. Then, it decreases in the transition regime of boiling up to a minimal value denoted $k_{\text {cld }}^{\min }$. This behaviour is specified in the nucleate boiling regime by the following relation:

$$
k_{c l l d}\left(T_{w}\right)=\frac{\alpha_{d r y}\left(T_{w}\right)}{\alpha_{d r y}^{P N}}
$$

where $\alpha_{d r y}$ is given by Equation (3) and $\alpha_{d r y}^{P N}=\alpha_{d r y}\left(T_{w}=T_{N}\right)$. 


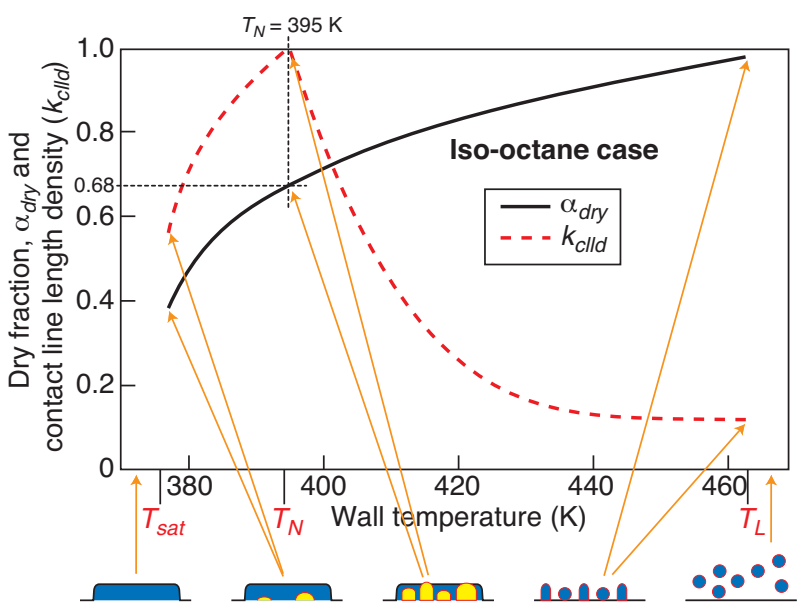

Figure 3

The function of dry fraction $\alpha_{d r y}$ and the function of length density of contact lines $k_{\text {clld }}$ drawn in the iso-octane case at atmospheric pressure. The schematics illustrate the boiling states near the limits of the boiling regimes.

In the transition boiling regime, $k_{\text {clld }}$ is supposed to decrease quickly towards $k_{\text {cld }}^{\mathrm{min}}$. This behavior is specified by the following expression:

$$
k_{\text {clld }}\left(T_{w}\right)=\left(1-k_{\text {clld }}^{\min }\right) T_{* *}^{4}+k_{\text {clld }}^{\min }
$$

where:

$$
T_{* * *}=\frac{T_{L}-T_{w}}{T_{L}-T_{s a t}}
$$

The $k_{\text {clld }}$ function (Eq. 6 and 7 ) is drawn in Figure 3 along with the $\alpha_{d r y}$ function ( $\left.E q .3\right)$ using data of iso-octane (Tab. 1). The diagrams in the lower part of this figure show the physical behaviours assumed to take place at the limits of the boiling modes, in particular close to the critical points of saturation, of Nukiyama and of Leidenfrost. The value of $k_{\text {cll }}^{\text {min }}$ is estimated according to surface roughness in the following paragraph.

\subsubsection{Influence of the Surface Roughness on Boiling}

Most of previous works on this issue (for instance those of Bernardin et al. [3] and Fardad and Ladommatos [9]) have reported that surface features promote liquid-wall contact and decrease droplet lifetime during the Leidenfrost regime. This suggests that rough surfaces require a thicker vapor cushion between the droplet and the surface to sustain film boiling. Thus, the influence of the average roughness of the wall $R u$ has been introduced into the LFB model via the value of $k_{\text {cll }}^{\min }$ in Equation (7). For perfectly smooth walls, we assumed a total levitation of the liquid film above a vapor cushion at the Leidenfrost point. This assumption leads to $k_{c l d}^{\mathrm{min}}=0$ when
$T_{w}=T_{L}$. For rough surfaces, the observed increase of liquidwall heat transfer has been modelled by setting:

$$
k_{\text {clld }}^{\min }=\frac{k_{R u 1}}{\alpha_{d r y}^{P N}} R u^{k_{R u 2}}
$$

where $k_{R u 1}$ and $k_{R u 2}$ are two constants to be adjusted using experimental data (cf. Sect. 3.2).

\subsubsection{Influence of the Gas Pressure on Boiling}

The gas pressure is an important parameter in the evaporation of liquid films as we underlined above in the bibliographical review (cf. for instance [9] and [23]). The increase in the pressure of gas induces two main effects:

\section{1st gas pressure effect}

Temple-Pediani [23] noted the absence of the vapor cushion which is the main characteristic of the Leidenfrost regime, if the gas pressure exceeds the critical pressure of the fluid. He explained the absence of the vapor cushion by the fact that the vapor pressure becomes insufficient to raise the liquid far from the wall. In other words, an increase in the gas pressure leads to a reduction of the rate of vaporization. In the LFB model, the reduction of the thickness of the vapor cushion as a function of the gas pressure is expressed by the following relationship:

$$
\delta_{v}=k_{\text {pres }}\left(\frac{p_{0}}{p}\right)^{2}
$$

where $p_{0}$ is taken equal to the atmospheric pressure ( 1 bar $)$, $p$ is the ambient pressure of gas, and $k_{\text {pres }}$ is the thickness of the vapor cushion when $p=p_{0}$. Its value shall come from available experimental results ( $c f$. Sect. 3.2). The square exponent of the pressure ratio was selected empirically using a fitting process ensuring a decrease of the vapor cushion thickness $\delta_{v}$ slower than the growth of the gas pressure in accordance with the experimental tendencies (cf. Sect.4.3).

\section{2nd gas pressure effect}

A shift of the lifetime curve towards the right side (i.e. towards the high wall temperatures) is observed when the gas pressure is increased. This shift involves $T_{s a t}, T_{N}$ and $T_{L}$ at the same time. On the one hand, the variation of the saturation temperature with pressure can be easily obtained using the Clausius-Clapeyron formula, at least for single-component liquid film. On the other hand, the shifts of $T_{N}$ and $T_{L}$ must be formulated according to the experimental observations available. This is going to be discussed in the following paragraph.

\subsection{Estimation of $T_{N}$ and $T_{L}$ Temperatures}

On the contrary of the saturation temperature, $T_{s a t}$, the temperatures of Nukiyama $T_{N}$ and Leidenfrost $T_{L}$ are not thermodynamic temperatures. Let us recall that $T_{N}$ corresponds to the point of maximum heat flux (CHF) or to the 
minimum point of the lifetime duration; and $T_{L}$ corresponds to local minimum of the curve of the heat flux or to the maximum of the lifetime duration curve separating the regimes of Leidenfrost and transition boiling, as shown in Figure 1. As indicated above, these critical temperatures depend on several factors $[9,11]$ including the properties of the wall (roughness, presence of impurity, wettability, thermal conductivity, specific heat, etc.) as well as the gravity, the gas pressure, the liquid flow rate, etc. One can find in the articles of Bernardin et al. [4-6] a complementary bibliographical review on this subject. In fact, previous research works have generally ignored the effects of the gas pressure on the $T_{N}$ Temperature and the available Leidenfrost temperature models have not met reasonable success. Moreover, few theoretical works dealt with the evaluation of the $T_{L}$ temperatures at high pressures. First, let us recall the low pressure $\left(P / P_{c}<<1\right)$ metastable liquid model of Speigler et al. [24] in which they correlated the critical temperature to the limiting temperature of homogeneous nucleation of an overheated liquid, assumed close to the $T_{L}$ temperature, as follows:

$$
T_{L}=\frac{27}{32} T_{c}
$$

In addition, for conditions of high pressures up to the critical pressure, Lienhard [25] suggested the following correlation:

$$
T_{L}=T_{c}\left[0.905+0.095\left(\frac{T_{s a t}}{T_{c}}\right)^{8}\right]
$$

This relationship describes the convergence of the point of Leidenfrost towards the critical temperature. Indeed, this behaviour was observed in experiments by Emerson and Snoek [26] who studied the effects of the pressure on the Leidenfrost point using Freon and water droplets. They showed that when the pressure increases, the Leidenfrost point of water on brass grows up to a value higher than the critical temperature, and then it decreases until the critical temperature when the pressure reaches the critical pressure. This tendency was confirmed experimentally by Breuer $e t a l$.
[27]. These authors showed that the temperature of Leidenfrost increases with the gas pressure. Moreover, it becomes equal to the saturation temperature as soon as the pressure exceeds half of the critical pressure.

Another interesting experimental work was published recently by Fardad and Ladommatos [9]. They showed that $\left(T_{L}-T_{N}\right)$ is very close to $\left(T_{N}-T_{s a t}\right)$ for several liquids including a gasoline and Diesel. Moreover, the differences $\left(T_{L}-T_{\text {sat }}\right)$ and $\left(T_{N}-T_{\text {sat }}\right)$ decrease when the gas pressure increases. In addition, they seem to be constant when the gas pressure decreases under the atmospheric pressure. This behavior are formulated by the following expressions:

$$
T_{c r}=T_{s a t}+\Delta T
$$

where $T_{c r}$ represents either $T_{N}$ or $T_{L}$ and $\Delta T$ is calculated according to the gas pressure $P$ in the following way :

$$
\Delta T= \begin{cases}T_{c r \mid \mathrm{bar}}-T_{b} & \text { if } P \leq 1 \mathrm{bar} \\ \frac{\left(T_{c r \mid 1 \mathrm{bar}}-T_{b}\right)-A}{T_{c}-T_{b}}\left(T_{c}-T_{s a t}\right)+A & \text { if } P>1 \mathrm{bar}\end{cases}
$$

where $T_{b}$ and $T_{c}$ are respectively, the normal boiling temperature and the critical temperature. For the high pressure case, the value of $\Delta T$ is assumed to tend linearly towards the value $A=\operatorname{Max}\left(1, T_{c r \mid 1 \mathrm{bar}}-T_{c}\right)$ when the gas pressure tends towards the critical pressure. Finally, let us note that the values of $T_{c r \mid P=1 \text { bar }}$ (i.e. $T_{N}$ and $T_{L}$ at $P=1$ bar) are supposed to be provided experimentally by lifetime curves of fuel droplets. Table 1 gathers the whole of the $T_{N}$ and $T_{L}$ data which we have found in the literature for fuels at atmospheric pressure. Whenever experimental values are not available, $T_{L 11 \text { bar }}$ may be estimated using Equation (11) but this may lead to a poor accuracy, and $T_{N 11 \text { bar }}$ could be estimated as follows:

$$
T_{N}=\frac{T_{b}+T_{L}}{2}
$$

\begin{tabular}{|c|c|c|c|}
\hline Fuels & $T_{N}(\mathrm{~K})$ & $T_{L}(\mathrm{~K})$ & References and experimental conditions \\
\hline$n$-pentane & 325 & 390 & \multirow{3}{*}{ [10]: Impact of drops $\left(d_{0}=2 \mathrm{~mm}\right)$ on aluminum heated plate } \\
\hline iso-octane & 395 & 463 & \\
\hline$n$-decane & 473 & 526 & \\
\hline$n$-heptane & 423 & 483 & $\begin{array}{l}\text { [9]: Impact of drops }\left(d_{0}=2.34 \mathrm{~mm}\right) \text { on aluminum heated plate. Average } \\
\text { Roughness } R u=0.22\end{array}$ \\
\hline Diesel & 643 & 733 & [9]: Impact of drops on aluminum heated plate. Average roughness $R u=0.22$ \\
\hline Diesel & 629 & 708 & [13]: Impact of drops ( $d_{0}: 0.19$ to $0.42 \mathrm{~mm}$ ) on stainless steel heated plate \\
\hline
\end{tabular}

TABLE 1

Nukiyama and Leidenfrost Temperatures obtained at atmospheric pressure. The experimental conditions given in the original papers are also recalled 


\subsection{Equations for the Vaporized Mass and the Wall Heat Flux}

The vaporized mass per unit area is calculated by the following energy balance:

$$
\dot{m}_{v}=\frac{\beta_{1}\left(1-\alpha_{d r y}\right) Q_{w l}+\beta_{2} \alpha_{d r y} Q_{w v l}}{L_{s a t}}
$$

where $L_{s a t}$ is the fuel latent heat of vaporization at the temperature $T_{s a t} . Q_{w l}$ is the heat flux per unit area between the wall and the liquid in direct contact. $Q_{w v l}$ is the heat flux per unit area between the wall and the liquid through the vapor cushion. This last becomes dominant compared to the first when the temperature of the wall tends towards the Leidenfrost temperature. On the one hand, $Q_{w l}$ is calculated by the following relation:

$$
Q_{w l}=\lambda_{l, s a t} \frac{\left(T_{w}-T_{s a t}\right)}{\delta_{t h}}
$$

where $\lambda_{l, \text { sat }}$ is the thermal conductivity of fuel at the temperature $T_{s a t}$ and $\delta_{t h}$ is a characteristic length of order of the thickness of the thermal boundary layer in the liquid film near the wall. $\delta_{t h}$ is the most important dependent parameter and is defined in Section 3.2. On the other hand, $Q_{w v l}$ is calculated by Equation (2) with a thickness of vapor cushion given by Equation (10).

In Equation (16), the $\beta_{1}$ parameter represents the fraction of the wetted area $\left(1-\alpha_{\text {dry }}\right)$ where most liquid vaporization is produced. In order to estimate $\beta_{1}$, let us recall that the vaporization of a liquid film in boiling modes occurs in a preferential way by an area band along the contact lines. At the CHF point, this area band around the film is proportional to $C_{\text {lld }}^{\max } \cdot h_{f}$ if the contact angle $\theta$ is equal to 90 degrees. Hence, the coefficient of proportionality $\beta_{1}$ mainly depends of $\theta$. In addition, by assuming that $\beta_{1}$ is proportional to the square of $k_{\text {clld }}$, this parameter can be written in the following way:

$$
\beta_{1}=C_{\text {lld }}^{\max } \frac{h_{f}}{\sin (\theta)} k_{\text {clld }}^{2}
$$

The second parameter $\beta_{2}$ in Equation (16) represents the fraction of dry area $\alpha_{d r y}$ where the liquid film is separated from the wall by a vapor cushion. As the theoretical determination of $\beta_{2}$ is difficult, it will be regarded as an adjustable parameter of the LFB model (see Sect. 3.2).

\section{VALIDATION OF THE LFB MODEL}

The determination of the parameters of the LFB model and its validation were carried out mainly on the basis of experimental data resulting from the articles of Stanglmaier et al. [10] and Fardad and Ladommatos [9]. In these experiments, the lifetime durations of rather bulky droplets $\left(d_{0}>2 \mathrm{~mm}\right)$ deposited on a hot surface were measured under various conditions. On the one hand, we retained from the article of Stanglmaier et al. [10] the lifetime duration curves that were measured using droplets of $n$-pentane, $n$-decane and iso-octane. For this last hydrocarbon, lifetime curves obtained using several ambient pressures are also available. On the other hand, we retained from [9], the lifetime curves of droplets of $n$-heptane deposited on surfaces of different average roughnesses.

\subsection{Initialization Method of the Liquid Film}

Once deposited gently on the wall, a droplet spreads and forms a liquid film of a maximum diameter (denoted $D_{\max }$ ) up to 3 to 5 times the initial diameter $d_{0}$ depending on the value of the droplet Weber number [9, 28, 29]. In this work, we assumed:

- The droplet after impact takes the form of a cylinder with radius $\overline{r_{f}}$ and height $\bar{h}_{f}$.

- The vaporized mass before the droplet takes the cylindrical form is negligible relatively to the mass of the droplet.

In order to calculate $\overline{r_{f}}$ and $\overline{h_{f}}$, we first used the model of Nagaoka et al. [30] which can be summarized by the following system of equations:

$$
\begin{aligned}
& r_{f}^{3}=\frac{4 r_{0}^{3} \sin ^{3} \phi}{2-3 \cos \phi+\cos ^{3} \phi} \\
& \cos \phi=\frac{1-\left(h_{h \max } / r_{f}\right)^{2}}{1+\left(h_{h \max } / r_{f}\right)^{2}} \\
& \frac{h_{h \max }}{r_{f}}=\frac{0.0354}{E_{0}^{0.51}} \text { and } E_{0}=\frac{4 g\left(\rho_{f}-\rho_{g}\right)}{\sigma_{f}}
\end{aligned}
$$

while denoting $r_{0}=d_{0} / 2$ and $h_{f \max }$ the height of the cylinder which has a radius $r_{f}=D_{\max } / 2$ (see the diagram of Fig. 4 for the notations). Then, one can evaluate the minimal height by:

$$
h_{f \min }=\frac{V_{0}}{\pi r_{f}^{2}} \quad \text { with } \quad V_{0}=\frac{4}{3} \pi r_{0}^{3}
$$

Finally, $\bar{h}_{f}$ and $\overline{r_{f}}$ are obtained by:

$$
\begin{aligned}
& \bar{h}_{f}={\frac{h_{f \max }+h_{f \min }}{2}}^{2} \\
& \bar{r}_{f}={\sqrt{\frac{V_{0}}{\pi \bar{h}_{f}}}}
\end{aligned}
$$

In Table 2, we summarise the initial characteristics of liquid films corresponding to the droplets used by Stanglmaier et al. [10] for $n$-pentane, iso-octane and 

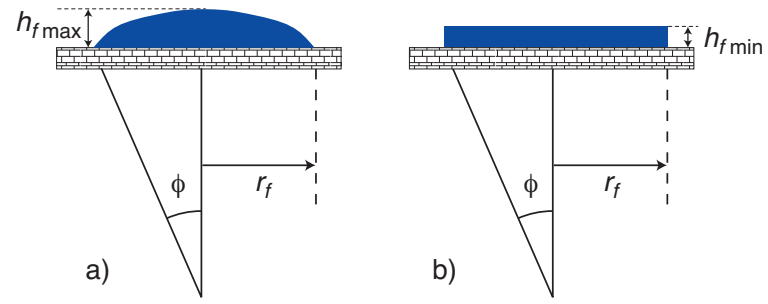

Figure 4

Numerical initialization of a droplet deposited on a wall. a) Schematic and notations for Nagaoka model [30]. b) Minimal film thickness, $h_{f \min }$ definition.

$n$-decane, and by Fardad and Ladommatos [9] for $n$-heptane. The ratio of the maximum spreading radius to the initial droplet radius just prior to impact, $\overline{r_{f}} / r_{0}$ is more or less equal to 4 . This large value may indicate that Stanglmaier et al. [10] and Fardad and Ladommatos [9] have probably used similar and relatively large Weber numbers [28]. The experimental Weber numbers were not given in their articles.

\section{TABLE 2}

Initial conditions for the calculations of lifetime durations for 4 liquid fuel droplets using the LFB model

\begin{tabular}{l|c|c|c|c}
\hline & $n$-pentane & iso-octane & $n$-decane & heptane \\
\hline$V_{0}(\mu \mathrm{L})$ & 5 & 5 & 5 & 6.7 \\
$r_{0}(\mathrm{~mm})$ & 1 & 1 & 1 & 1.17 \\
$\bar{r}_{f} / r_{0}$ & 4.1 & 4.28 & 4.32 & 4.1 \\
$A_{f}=\pi r_{f}^{-2}\left(\mathrm{~mm}^{2}\right)$ & 53.3 & 57.4 & 58.7 & 72.3 \\
$\bar{h}_{f}(\mu \mathrm{m})$ & 94 & 87 & 85 & 93 \\
\hline
\end{tabular}

\subsection{Choice of the LFB Model Parameters}

The parameters of LFB model which remain to be determined are: $\delta_{t h}, \beta_{2}, k_{R u 1}, k_{R u 2}$ and $k_{\text {pres }}$.

\section{$\delta_{t h}$ estimation}

$\delta_{t h}$ is a characteristic length for the calculation of the heat flux between the wall and the liquid in direct contact (Eq. 17). Its value is of the order of the thickness of the thermal boundary layer in the liquid on the wall. It depends mainly on the thermo-physical properties of the liquid. For the estimation of $\delta_{t h}$, we considered that the Critical Heat Flux (CHF) comes primarily from the heat flux between the wall and the liquid in direct contact. Indeed, Temple-Pediani [10] showed that the heat flux dominating is that which comes from the wall by direct contact with the liquid, especially near the CHF. Under these conditions, the vaporization rate $\dot{m}_{C H F}$ at $T_{w}=T_{N}$ can be written as follows:

$$
\dot{m}_{C H F}=\frac{\beta_{1}\left(1-\alpha_{d r y}\right)}{L_{s a t}} \frac{\lambda_{l, s a t}\left(T_{N}-T_{s a t}\right) A_{f}}{\delta_{t h}}
$$

In addition, Stanglmaier et al. [10] has provided an estimation of this vaporization rate: $\dot{m}_{C H F} \approx 20 \mathrm{mg} / \mathrm{s}$ in the case of iso-octane. Moreover, Equation (3) gives $\alpha_{d r y} \approx 0.68$ and $k_{\text {clld }}=1$ at $T_{w}=T_{N}$ (Fig. 3). Then, using Equation (18) and assuming an average contact angle $\theta$ equal to 150 degrees [29, 31], one can obtain $\beta_{1} \approx 0.5$. We finally used the physical properties of iso-octane at atmospheric pressure (Tab. 3) in Equation (21) to obtain $\delta_{t h} \approx 3 \mu \mathrm{m}$. In practice, we used $\delta_{t h}=\min \left(h_{f}, 3 \mu \mathrm{m}\right)$ to remain coherent with film thicknesses lower than $3 \mu \mathrm{m}$.

TABLE 3

Physical parameters of iso-octane at atmospheric pressure used to calculate $\delta_{t h}$ using Equation (21)

\begin{tabular}{c|c|c|c}
\hline$\lambda_{l, s a t}(\mathrm{~W} /(\mathrm{m} . \mathrm{K}))$ & $L_{\text {sat }}(\mathrm{J} / \mathrm{kg})$ & $T_{\text {sat }}(\mathrm{K})$ & $T_{N}(\mathrm{~K})$ \\
0.1 & $2.67 \times 10^{5}$ & 372 & 395 \\
\hline
\end{tabular}

\section{$\beta_{2}$ estimation}

$\beta_{2}$ is the fraction of $\alpha_{d r y}$ where the liquid film is separated from the wall by a vapor cushion in Equation (16). This means that $\left(1-\beta_{2}\right) \alpha_{d r y}$ is the fraction of the initial liquid film area $A_{f}$ which is already completely dry. In this work, $\beta_{2}$ is supposed to be constant during the boiling transition regime. We adjusted its value on the experimental lifetime curve duration of iso-octane at atmospheric pressure from Stanglmaier et al. [10]. The value $\beta_{2}=0.055$ gives the best fit as shown in Figure 5.

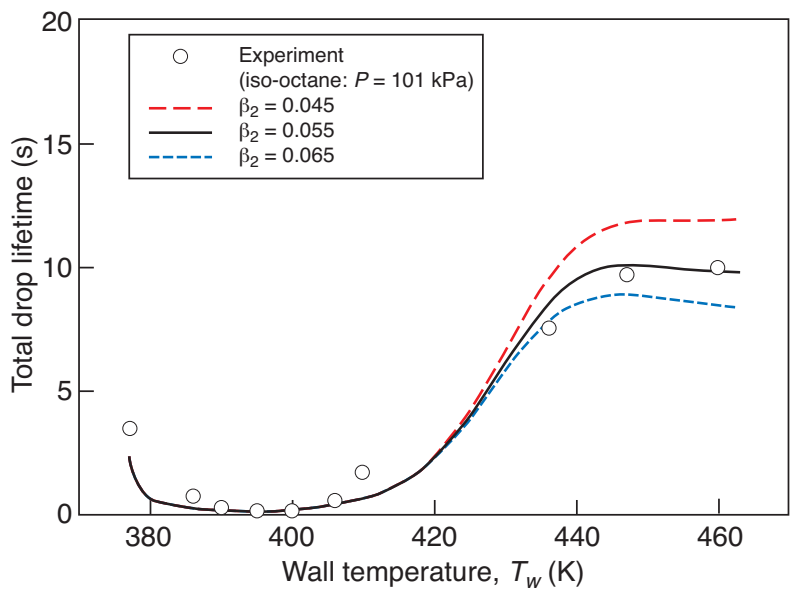

Figure 5

Results for $\beta_{2}$ parameter adjustment using the curve of experimental lifetime of iso-octane. The experimental data was taken from [10]. 


\section{$k_{R u 1}$ and $k_{R u 2}$ estimation}

They are the two parameters in Equation (9) relating the dimensionless function of length density of contact lines $k_{\text {clld }}$ to the average roughness of the wall. For the determination of these two coefficients, we used, once again the experimental curve of lifetime duration of the iso-octane droplet. We proceeded by trial and error and by supposing that the aluminium block on which Stanglmaier et al. [10] deposited the droplets is relatively smooth $(R u=0.2 \mu \mathrm{m})$. The results presented in Figure 5 were obtained using $k_{R u 1}=1$ and $k_{R u 2}=0.2$.

\section{$\mathbf{k}_{\text {pres }}$ estimation}

$k_{\text {pres }}$ is a characteristic thickness of the vapor cushion $\delta_{v}$ separating the liquid from the wall in the Leidenfrost regime (see Eq. 2). Actually, few theoretical works have been devoted to the effect of pressure on the thickness of the vapor cushion $\delta_{v}$ in the Leidenfrost regime. Using a theoretical model, Rein [1] showed that the characteristic value of the thickness of the vapor cushion in the Leidenfrost regime is between 10 and $100 \mu \mathrm{m}$. In the same way, Yang and Fan [32] have recently developed a numerical model for the simulation of the impact of a droplet on a plane plate in the Leidenfrost regime. They calculated the profile thickness of the vapor cushion during the impact. They found in particular that the value of $\delta_{v}$ varies during the impact between $120 \mu \mathrm{m}$ and $10 \mu \mathrm{m}$. In addition, the experiments of Chandra and Aziz [33] and Kistemaker [34] do corroborate this order of magnitude. Thus, we considered in the LFB model, $\delta_{v}=100 \mu \mathrm{m}$ for a pressure $p_{0}=1 \mathrm{bar}$. This value leads to $k_{\text {pres }}=100 \mu \mathrm{m}$ in Equation (10). For a supercritical gas ambient pressure (saying for a fuel, $p=20$ bars),
Equation (10) gives $\delta_{v}=0.25 \mu \mathrm{m}$. This small value is in conformity with the experimental observations of TemplePediani [23].

\subsection{Sensitivity of LFB Model to the Values of $T_{N}$ and $T_{L}$}

We already presented in Section 2.3 Equations (13) and (14) following the estimation of the temperatures of Nukiyama $T_{N}$ and Leidenfrost $T_{L}$ according to the ambient pressure value $P$. The experimental values of these temperatures are not available for all fuels. In the following two subsections, the sensitivity of the results of the LFB model to the given values to these two temperatures is studied.

\subsubsection{Sensitivity to the $T_{N}$ Value}

Two simulations were carried out with the LFB model in the case of an iso-octane droplet at atmospheric pressure. The first simulation uses the experimental value of the temperature of Nukiyama $\left(T_{N, \exp }=395 \mathrm{~K}\right)$ and the second uses the following estimation: $T_{N}=\left(T_{\text {sat }}+T_{L, \text { exp }}\right) / 2=419 \mathrm{~K}$, as shown in Figure 6. One can see that the increase by $6 \%$ of the value of $T_{N}$ results in a delaying of the nucleate boiling and the transition boiling regimes. For the latter regime, this delaying has led to an overestimation of the vaporized mass.

\subsubsection{Sensitivity to the $T_{L}$ Value}

In the same way as described previously, two simulations were carried out with the LFB model in the case of a $n$-heptane droplet at atmospheric pressure. The first simulation uses the experimental value of the temperature of Leidenfrost $\left(T_{L, \exp }=483 \mathrm{~K}\right)$ and the second uses $T_{L}=456 \mathrm{~K}$

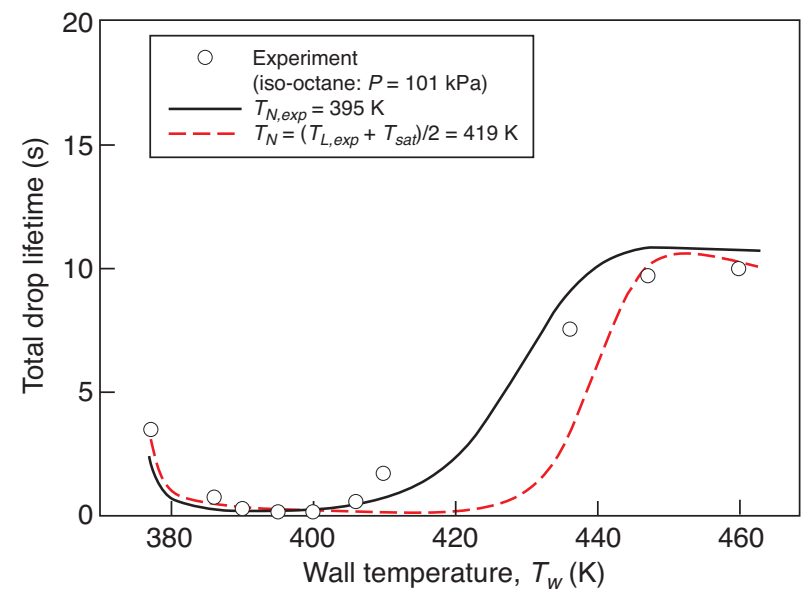

Figure 6

Sensitivity of the results of LFB model according to the value of the temperature of Nukiyama $T_{N}$ in the case of an isooctane droplet at atmospheric pressure. The experimental data was taken from [10].

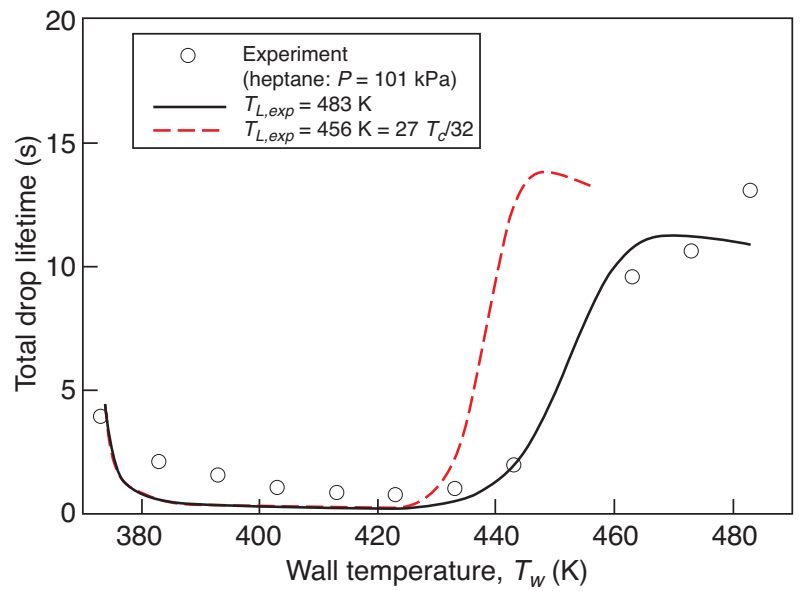

Figure 7

Sensitivity of the results of LFB model according to the value of the temperature of Leidenfrost $T_{L}$ in the case of a $n$ heptane droplet at atmospheric pressure. The dashed curve was obtained using Equation (11). The experimental data was taken from Fardad and Ladommatos [9]. 


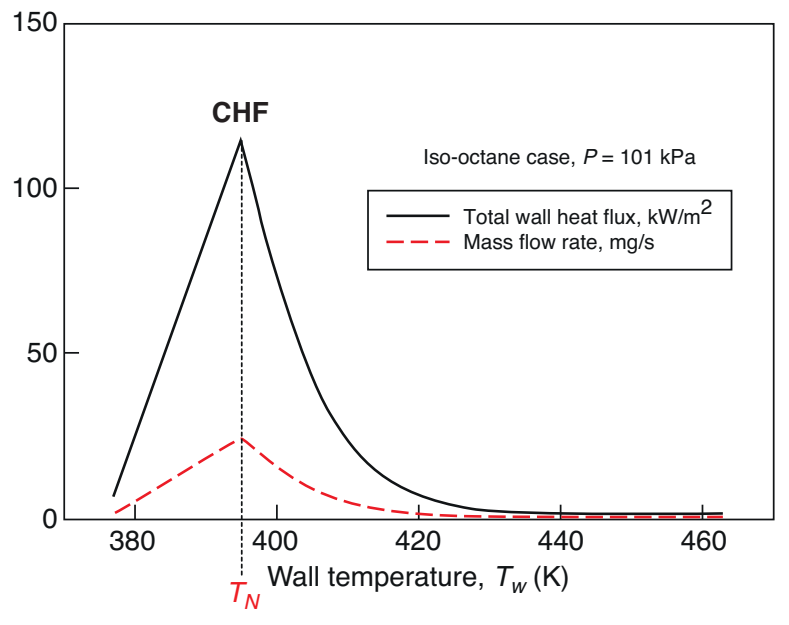

Figure 8

Total heat flux given by the wall to the liquid film (solid line) and vaporized mass (dashed line) curves in the case of an isooctane droplet with atmospheric pressure.

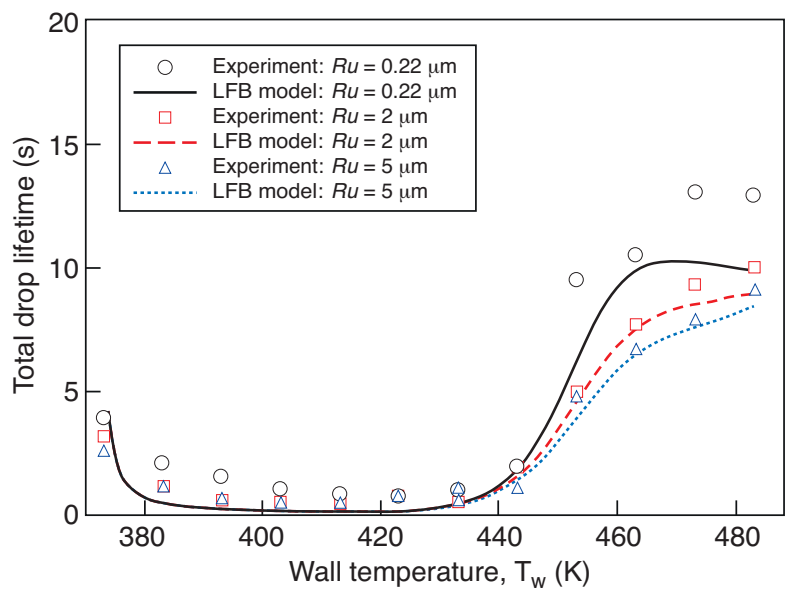

Figure 10

Influence of surface roughness on the lifetime of an isooctane droplet. Comparison of the LFB results with the measurements of Fardad and Ladommatos [9].

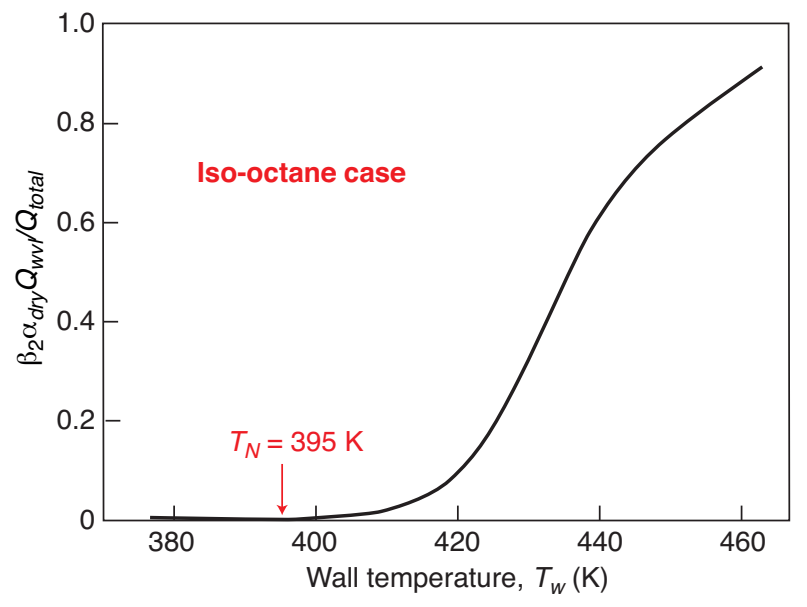

Figure 9

Fraction of the total heat flux that passes through the vapor cushion during the nucleate and transition boiling modes. Results obtained in the case of iso-octane with atmospheric pressure.

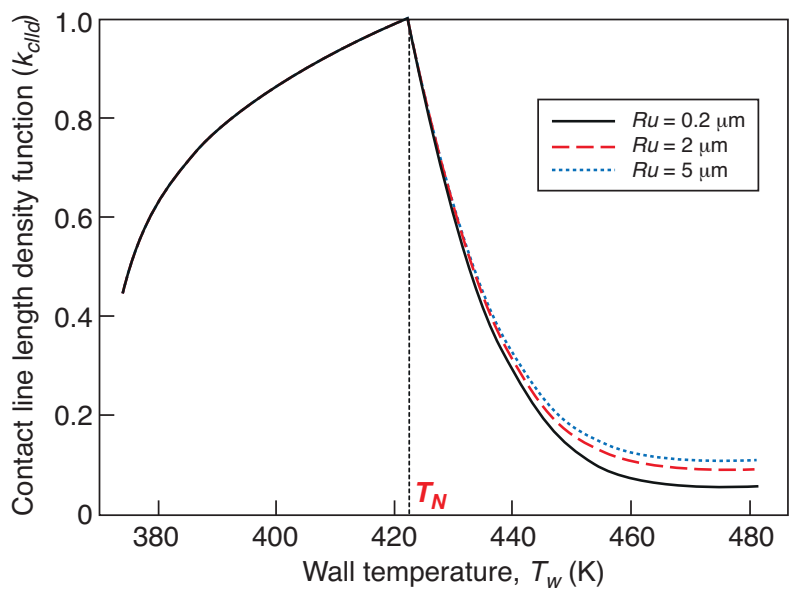

Figure 11

Influence of the roughness on the function of contact lines length density $k_{\text {clld }}\left(T_{w}\right)$. obtained using Equation (11). This equation results in an underestimation of more than $5 \%$ on $T_{L}$ values. This error leads to a shortening of the transition boiling regime due to an underestimation of the vaporized mass as shown in Figure 7.

\section{DISCUSSION OF THE RESULTS OF THE LFB MODEL}

Initially, let us consider again the case of the iso-octane droplet studied in experiments by Stanglmaier et al. [10]. Figure 8 shows the curves of total wall-liquid heat flux and of vaporized mass obtained by the LFB model in the atmos- pheric pressure case. It is worth noting that the maximum vaporized mass ( $\sim 20 \mathrm{mg} / \mathrm{s})$ obtained at the Critical Heat Flux (CHF) is obviously in a satisfactory agreement with the experimental value of Stanglmaier et al. [10]. Moreover, the order of magnitude of the $\mathrm{CHF}$ is more or less equal to $120 \mathrm{~kW} / \mathrm{m}^{2}$. This value is in line with the experimental observations met in the open literature.

In addition to the classical heat flux in the wetted wall zone, the main characteristic of the LFB model lies in the explicit consideration of the heat flux between the wall and the liquid through the vapor cushion. Figure 9 shows that 


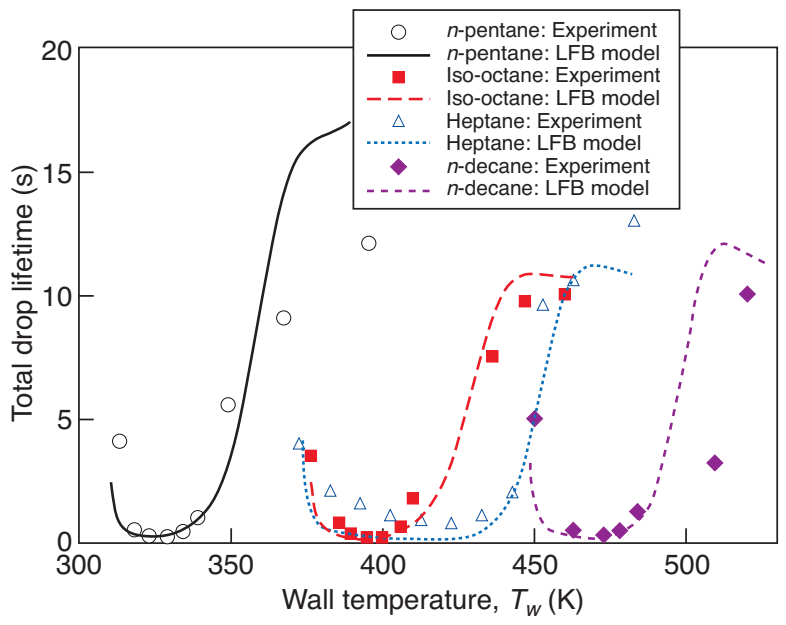

Figure 12

Comparisons of the LFB model results with the measurements of the lifetimes of droplets with atmospheric pressure for 4 pure fuels: $n$-pentane, iso-octane, $n$-heptane and $n$-decane. The experimental data was taken from $[9,10]$.

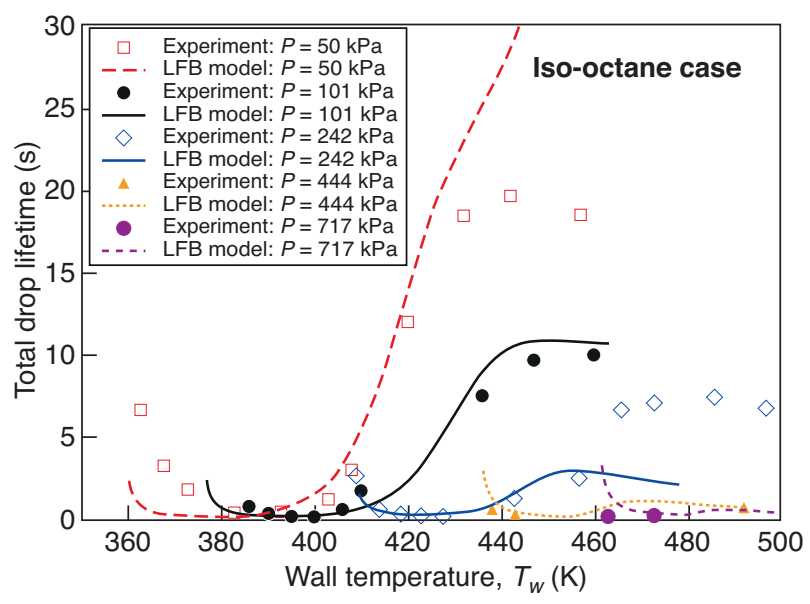

Figure 13

Influence of ambient gas pressure $P$ on the lifetime of an isooctane droplet. Comparison of the LFB model results with the measurements of Stanglmaier et al. [10]. the percentage of this last heat flux increases during the transition boiling regime, in a nonlinear way up to $90 \%$ of the total heat flux $\left(Q_{\text {total }}=m_{v} L_{s a t}\right)$ at the Leidenfrost point. In fact, the value of this percentage at the Leidenfrost point depends on a multitude of factors among which roughness that we are going to study in the following paragraph.

\subsection{Influence of the Surface Roughness}

We used here the experimental results of Fardad and Ladommatos [9]. These results depicted again in Figure 10, show a correct sensitivity of the numerical results of the LFB model with the average roughness of the wall. Indeed, the numerical results show a gradual increase of the vaporization rate before the Leidenfrost point as the wall roughness is increased, in accordance with the Fardad and Ladommatos [9] experiments. However, the model overestimates the vaporization rate increase slightly for small roughnesses which leads to the presence of a plateau before the point of Leidenfrost. In addition, the LFB model does not reproduce the effect of wall roughness observed in the nucleate boiling zone.

Besides, Figure 11 shows the evolution of the dimensionless function $k_{\text {clld }}$ expressed by Equations (6), (7) and (9). One may note that in the LFB model roughness increases the contact of the liquid with the wall near the Leidenfrost point, in accordance with the experimental observations [7,9].

\subsection{Influence of the Fuel Properties}

We carried out here calculations using the LFB model for the 4 following fuels: $n$-pentane, iso-octane, heptane and the $n$-decane at atmospheric pressure. The curves of droplet lifetime obtained numerically are compared with experiments in Figure 12. The LFB model correctly renders the general $S$ shape of the curves of lifetime in both nucleate and transition regimes of boiling. The minimal value of the droplet lifetime durations is in agreement with the measurements for all the fuels tested. In addition, the LFB model qualitatively reproduces the experimental observations in the zone of passage from the transition boiling regime towards the Leidenfrost regime. The orders of magnitude of the lifetime durations at Leidenfrost point are also correctly predicted.

\subsection{Influence of the Gas Pressure}

A second series of calculations were carried out to test the response of the LFB model to the pressure of ambient gas (see Sect 2.2.2). Figure 13 compares the numerical results with the experiments of Stanglmaier et al. [10]. One may note that the dependence of the temperatures of Nukiyama and Leidenfrost with the pressure (Eq.13 and 14 ) is correctly predicted. In addition, the LFB model reproduces well the progressive disappearance of the regime of Leidenfrost observed in experiments $[10,23]$. It is however worth noting some dissension between the experimental and numerical curves, in particular at the beginning of the nucleate boiling regime (with low pressure, $P=50 \mathrm{kPa}$ ) and towards the end of the transition boiling regime (with moderated pressure, $P=242 \mathrm{kPa}$ ). These dissensions may be due to both experimental and numerical uncertainties; for example, some uncertainties could arise from the approximations made in 
the method of initialization of the numerical liquid film on the wall (see Sect. 3.1). Nevertheless, the LFB model seems to behave correctly and the orders of magnitude and the tendencies observed experimentally are well respected.

\section{CONCLUSIONS}

A comprehensive Liquid Film Boiling (LFB) model has been developed using available experimental results from the literature. The main characteristics of the LFB model are:

It allows the calculation of the vaporization of liquid films in the nucleate boiling regime as well as in the transition boiling mode. These modes are identified by the temperatures of saturation, Nukiyama and Leidenfrost.

It takes into account the effects of the ambient gas pressure as well as the wall roughness on the evaporation rate of liquid films.

Relationships representing the variation of the temperatures of Nukiyama and Leidenfrost with the gas pressure were proposed and validated. It is shown that values of these temperatures at ambient pressure must result from the experimental curves of droplets lifetimes in order to ensure a correct behaviour of the LFB model.

The numerical results of the LFB model compare well with the experiments under various conditions including the variations of the wall roughness and of the ambient gas pressures which are of considerable interest in several devices like internal combustion engines.

\section{REFERENCES}

1 Rein M. (2002) Interactions between drops and hot surfaces, in Drop-Surface Interactions, Springer-Verlag, pp. 185-217.

2 Chaves H., Kubitzek A.M., Obermeier F. (1999) Dynamic processes occurring during the spreading of thin liquid films produced by drop impact on hot walls, Int. J. Heat Fluid Fl. 20, 5, 470-476.

3 Bernardin J.D., Stebbins C.J., Mudawar I. (1996) Effects of surface roughness on water droplet impact history and heat transfer regimes, Int. J. Heat Mass Tran. 40, 1, 73-88.

4 Bernardin J.D., Stebbins C.J., Mudawar I. (1997) Mapping of impact and heat transfer regimes of water drops impinging on a polished surface, Int. J. Heat Mass Tran. 40, 247-267.

5 Bernardin J.D., Mudawar I. (1999) The Leidenfrost Point: Experimental Study and Assessment of Existing Models, J. Heat Trans-T. ASME 121, 894-903.

6 Bernardin J.D., Mudawar I. (2004) A Leidenfrost point model for impinging droplets and sprays, J. Heat Trans.-T. ASME 126, 2, 272-278.

7 Moita A.S., Moreira A.L.N. (2007) Drop impacts onto cold and heated rigid surfaces: Morphological comparisons, disintegration limits and secondary atomization, Int. J. Heat Fluid Fl. 28, 4, 735-752.

8 Desoutter G., Cuenot B., Habchi C., Poinsot T. (2005) Interaction of a premixed flame with a liquid fuel film on a wall, Proc. Combust. Inst. 30, 259-266.
9 Fardad D., Ladommatos N. (1999) Evaporation of hydrocarbon compounds, including gasoline and diesel fuel, on heated metal surfaces, Proc. Inst. Mech. Eng. Part D - J. Automobile Eng. 213, D6, 625-645.

10 Stanglmaier R.H., Roberts C.E., Moses C.A. (2002) Vaporization of Individual fuel drops on a heated surface: A study of fuel-wall interactions within Direct-injected Gazoline (DIG) Engines, SAE paper 2002-01-0838.

11 Dhir V.K. (1998) Boiling heat transfer, Annu. Rev. Fluid Mech. 30, 365-401.

12 Dhir V.K. (1991) Nucleate and transition boiling heat transfer under pool and external flow conditions, Int. J. Heat Fluid Fl. 12, 4, 290-314.

13 Xiong T.Y., Yuen M.C. (1991) Evaporation of a liquid droplet on hot plate, Int. J. Heat Mass Tran. 34, 7, 1881-1894.

14 Tamura Z., Tanasawa Y. (1959) Evaporation and Combustion of a drop contacting with a hot surface, Symp. Combust. 509-522.

15 Takano T., Fujita T., Kobayasi K. (1995) Vaporization Behavior of a Sigle Droplet Impinging on hot Surface with a Flame-Sprayed Ceramic Coating and a Pressurized Atmosphere, Heat Transfer- Jap. Res. 24, 1, 80-97.

16 O'Rourke P., Amsden A. (2000) A Spray/wall Interaction Submodel for the KIVA-3 Wall Film Model, SAE paper 2000-01-0271.

17 Desoutter G., Habchi C., Cuenot B., Poinsot T. (2006) Single-component Liquid film evaporation model development and validation using Direct Numerical Simulation, ICLASS Kyoto, 2006.

18 Desoutter G., Habchi C., Cuenot B., Poinsot T. (2009) DNS and modeling of the turbulent boundary layer over an evaporating liquid film, Int. J. Heat Mass Tran. doi:10.1016/j.ijheatmasstransfer.2009.06.039.

19 Iida Y., Kobayasi K. (1969) Distribution of void fraction above a horizontal heating surface in pool boiling, Bull. JSME 12, 283-290.

20 Nishio S., Tanaka H. (2002) Simplified Model Predicting Contact-Line-Length Density at Critical Heat Flux Based on Direct Observation of Boiling Structure, JSME Int. J. B-Fluid T. 50, 1, 72-78.

21 Dhir V.K., Liaw S.P. (1989) Framework for a Unified Model for Nucleate and Transition Pool Boiling, J. Heat Trans.-T. ASME 111, 739-746.

22 Nigmatulin B.I., Vasiliev N.I., Guguchkin V.V. (1993) Interaction between liquid droplets and heated surface, Warme Stoffubertragung 28, 313-319.

23 Temple-Pediani R.W. (1969) Fuel drop vaporization under pressure on a hot surface, Proc. Inst. Mech. Eng. 184 Pt. 1, 38, 677-696.

24 Spiegler P., Hopenfeld J., Silberberg M., Bumpus J., Norman A. (1963) Onset of stable film boiling and the foam limit, Int. J. Heat Mass Tran. 6, 11, 987-989.

25 Lienhard J.H. (1976) Correlation for the limiting liquid superheat, Chem. Eng. Sci.31, 9, 847-849.

26 Emmerson G.S., Snoek C.W. (1978) The effect of pressure on the leidenfrost point of discrete drops of water and freon on a brass surface, Int. J. Heat Mass Tran. 21, 8, 1081-1086.

27 Breuer A., Klingsporn M., Schneemann G.A., Wruck N., Renz U. (1993) Experimental and Theoritical Investigation of the Phase Transition of Multicomponent Sprays and the Influence of the wall heat flux, Periodic Report, 01.07.1993 to 31.12.1993, Contract JOU2-CT92-0162. 
28 Chen R.H., Chiu S.L., Lin T.F. (2207) On the collision behaviors of a diesel drop impinging on a hot surface, Exp. Therm. Fluid Sci. 32, 2, 587-595.

29 Kandlikar S.G., Steinke M.E. (2001) Contact angles of droplets during spread and recoil after impinging on a heated surface, Chem. Eng. Res. Des. 79, A4, 491-498.

30 Nagaoka M., Kawazoe H., Nomura N. (1994) Modeling Fuel Spray Impingement on a Hot Wall for Gasoline Engines, $S A E$ paper 940525.

31 Kandlikar S.G., Steinke M.E. (2002) Contact angles and interface behavior during rapid evaporation of liquid on a heated surface, Int. J. Heat Mass Tran. 45, 18, 3771-3780.
32 Yang G.E., Fan L.-S. (2005) Three-dimensional simulation of impingement of a liquid droplet on a flat surface in the Leidenfrost regime, Phys. Fluids 17, 027104, 1-20.

33 Chandra S., Aziz S.D. (1994) Leidenfrost Evaporation of Liquid Nitrgen Droplets, J. Heat Trans.-T. ASME 116, 9991006.

34 Kistemaker J. (1963) The spheroidal state of waterdrop, Physica 29, 96-104.

Final manuscript received in August 2009

Published online in April 2010 